\title{
Mortero geopolimérico para uso potencial como recubrimiento en concreto
}

\author{
KATHERINE OVIEDO-SÁNCHEZ ${ }^{1}$ \\ Ruby MEJÍA-DE-GuTIÉRREZ ${ }^{1}$
}

\section{Resumen}

Se sintetizaron dos morteros geopoliméricos basados en Metacaolín y residuo de ladrillo, modificados con adiciones orgánicas e inorgánicas para ser utilizados como potenciales recubrimientos sobre concretos. Se evaluaron sus propiedades en estado fresco (fluidez, tiempo de fraguado) y en estado endurecido (resistencia a la compresión y tracción, absorción, porosidad y conductividad térmica). Los resultados muestran la viabilidad de producir morteros Clase R1 y R2 en concordancia con la Norma EN 1504-3 en cuanto a las propiedades mecánicas y se evidencia que los sistemas geopoliméricos producidos presentan menor conductividad térmica comparada a los morteros basados en OPC sugiriendo comportamiento tipo aislante.

Palabras clave: Geopolímeros; Metacaolín; Polvo de Ladrillo; Recubrimientos

\section{Geopolymeric Mortar for Potential Use as a Concrete Coating}

\section{Abstract}

In the present study, two geopolimeric mortars based on Metakaolin and brick residue were synthesized; these were modified with organic and inorganic additions to be used as potential coatings on concrete. Properties in a fresh state (fluidity, setting time) and in a hardened state (compressive and traction strength, absorption, porosity and thermal conductivity) were evaluated. The results show the feasibility of producing Class R1 and R2 mortars in accordance with

1 Universidad del Valle, Cali, Colombia.

Autor de correspondencia: Mejía-de-Gutiérrez, R. (Ruby): Universidad del Valle: Cl. 13 No.100-00, Edificio 349, Cali, Colombia. Teléfono: (+57) 2 3302436. Correo electrónico: ruby.mejia@correounivalle.edu.co
Historia del artículo:

Artículo recibido: 10-V-2018/ Aprobado: 12-I-2019

Disponible online: 3 de febrero de 2019

Discusión abierta hasta octubre de 2020 
the EN 1504-3 Standard in terms of mechanical properties. The geopolymeric systems produced have a lower thermal conductivity compared to mortars based on OPC, which suggests its use as insulating coatings.

Keywords: Brick Powder; Coatings; Geopolymers; Metakaolin.

\section{Argamassa geopolimérica para uso potencial como revestimento de betão}

\section{Resumo}

No presente estudo, duas argamassas geopoliméricas baseadas em Metakaolin e resíduo de tijolo foram sintetizadas; estes foram modificados com adições orgânicas e inorgânicas para serem usados como revestimentos potenciais no concreto. Propriedades em estado fresco (fluidez, tempo de endurecimento) e em estado endurecido (resistência à compressão e tração, absorção, porosidade e condutividade térmica) foram avaliadas. Os resultados mostram a viabilidade de produzir argamassas da classe R1 e R2 de acordo com a norma EN 1504-3 em termos de propriedades mecânicas. Os sistemas geopoliméricos produzidos possuem menor condutividade térmica em relação às argamassas à base de OPC, 0 que sugere sua utilização como revestimento isolante.

Palavras-chave: Pó de Tijolo; Revestimentos; Geopolímeros; Metakaolin.

\section{Introducción}

A nivel mundial, la industria de la construcción se ha venido enfocando en mejorar y prolongar la vida útil de grandes estructuras, edificaciones y residencias producidas en concreto, debido a que la exposición de éstos en diferentes ambientes agresivos como lo son las zonas industriales y las zonas costeras generan perdida de la integridad del material con el paso del tiempo. Una vez se produce el daño en la estructura, repararlo puede ser muy costoso, difícil e imposible en algunos casos (Vejmelková et al., 2012), (Wiyono, Antoni and Hardjito, 2015). Por ejemplo, en Europa alrededor de 215 mil euros se destinan anualmente para el mantenimiento de puentes de hormigón. En los Estados Unidos en un promedio de 5 años, aproximadamente 1,6 billones de dólares se destinan a la reparación, donde $27 \%$ de ese dinero corresponde a la recuperación de puentes y carreteras, 150 mil millones de dóla- res para daños por corrosión y el resto para edificaciones e infraestructura de aguas residuales en las cuales se han estimado inversiones de hasta 21 mil millones de dólares anuales (Pacheco-Torgal et al., 2012). Las operaciones de mantenimiento frecuentes o de sustitución completa de una infraestructura, además del alto costo, se traducen en un aumento del impacto ambiental debido al nuevo consumo de materias primas, energía y servicios de transporte para su reparación (Pacheco-Torgal et al., 2012). Si bien es cierto, que, aunque estas reparaciones no involucran grandes cantidades de concreto en comparación a la fabricación de una nueva estructura, constantemente se rehabilitan puentes, edificios, carreteras incluso tuberías de aguas residuales, ya que son propensas a carbonatación, permeabilidad de cloruros y en el último caso a ataques por ácido sulfúrico producto de la oxidación del azufre mediante bacteria (Tamburini et al., 2017). 
Con el fin de disminuir el impacto ambiental y mejorar la durabilidad de los materiales de construcción una técnica es recurrir al uso de recubrimientos, entre los cuales los más empleados son los recubrimientos orgánicos a base de polímeros reforzados los cuales son de fácil aplicación, sin embargo, presentan algunas deficiencias como baja permeabilidad al vapor de agua, poca resistencia al fuego y al envejecimiento, y degradación por radiación UV, así como también un alto costo. Debido a esto se ha incrementado el estudio de nuevos materiales que presenten mejor desempeño como recubrimientos exteriores en estructuras de concreto, entre los cuales de uso reciente se encuentran los materiales geopoliméricos (Tamburini et al., 2017), (Zhang et al., 2015). Los morteros de recubrimiento a base de cementantes alternativos (geopolímeros) ha tenido gran acogida debido a que pueden ser sintetizados mediante activación alcalina de residuos industriales como las cenizas volantes, escorias siderúrgicas, polvo de ladrillo, lodos, residuos de demolición, entre otros, con mínima liberación adicional de dióxido de carbono $\mathrm{CO}_{2}$, y además han presentado excelente adhesión a otros materiales, buenas propiedades mecánicas, físicas, durabilidad química e inhibición al fuego, propiedades que en algunos casos superan las de materiales basados en el cemento Portland (Irfan Khan et al., 2015).

Irfan Khan et al., (2017) estudiaron la capacidad de adherencia y estabilidad termica, de un recubrimiento a base de ceniza volante (CV) tipo $\mathrm{F}$ activada con $\mathrm{NaOH}$, con relaciones $\mathrm{Si} / \mathrm{Al}=1.78, \mathrm{Na}$ / $\mathrm{Al}=1,0 \mathrm{y} \mathrm{L} / \mathrm{s}=0,33$ y reportan una fuerza de adhesión de 3,8 MPa y una perdida de masa de 11-12\% a $800^{\circ} \mathrm{C}$. Mu et al,. (2017) evaluaron un recubrimiento de $2 \mathrm{~mm}$ de espesor a base de metacaolín (MK) activado con una mezcla de $\mathrm{Na}_{2} \mathrm{SiO}_{3}$ y $\mathrm{NaOH}(12,5$ mol/l), con relaciones $\mathrm{SiO}_{2} / \mathrm{Na}_{2} \mathrm{O}$ y L/s de 0.8 y 0.9 , respectivamente y obtuvieron resistencia a la adherencia de 0,84 y 0,92 MPa a los 7 y 28 días. Krivenko et al., (2016) utilizó recubrimientos de MK con espesores de 2, 6 y $12 \mathrm{~mm}$ activados con una mezcla de $\mathrm{Na}_{2} \mathrm{SiO}_{3}$ y NaOH con relaciones $\mathrm{Na} / \mathrm{Al}=1, \mathrm{Si} / \mathrm{Al}=6$ $\mathrm{y}_{2} \mathrm{O} / \mathrm{Al}=20$, encontrando que al incrementar el espesor la adherencia disminuye. Zhang et al,. (2015) evaluo las propiedades aislantes y reflectivas de un recubrimiento basado en $\mathrm{MK}$ activado con $\mathrm{Na}_{2} \mathrm{SiO}_{3} \mathrm{y}$ adicionado con dióxido de titanio $\left(\mathrm{TiO}_{2}\right)$ y microesferas de vidrio huecas, e informan que el aislamiento térmico y la reflectividad mejora en $12 \%$ y $90 \%$ respectivamente, a la par que se obtiene una estructura densa con buenas propiedades de durabilidad, adherencia y bajo tiempo de fraguado. Resultados similares fueron encontrados por Pacheco-Torgal et al., (2012) y Liew et al., (2016), quienes mencionan el uso potencial de los geopolímeros en la rehabilitación de infraestructura y como materiales de sellado sustituyendo adhesivos epóxidos, siempre y cuando estén reforzados con fibras como las de polipropileno para controlar la contracción.

Teniendo en cuenta lo mencionado anteriormente, en el presente proyecto de investigación se pretende evaluar un mortero de recubrimiento geopolimérico a partir de dos tipos de precursores para su uso en superficies de concreto, que además de presentar buenas propiedades mecánicas, físicas y químicas permita también un buen acabado superficial con el fin de obtener calidad estética para edificaciones y residencias.

\section{Metodología experimental}

La metodología llevada a cabo es presentada en la Figura 1; esta consta de dos etapas, en la primera la elaboración y caracterización de morteros geopoliméricos aptos para su aplicación como recubrimientos y en una segunda etapa la validación del material como recubrimiento sobre sustratos de concreto. El presente artículo se limita a presentar los resultados obtenidos en la primera etapa del estudio, tomando en consideración algunos de los parámetros especificados de la norma europea EN 1504-3, la cual clasifica los tipos de recubrimiento y las características que estos deben cumplir para su aplicación en procesos de reparación de estructuras de concreto (Tabla 1). 
Figura 1. Diagrama de flujo de la metodología empleada en el proyecto

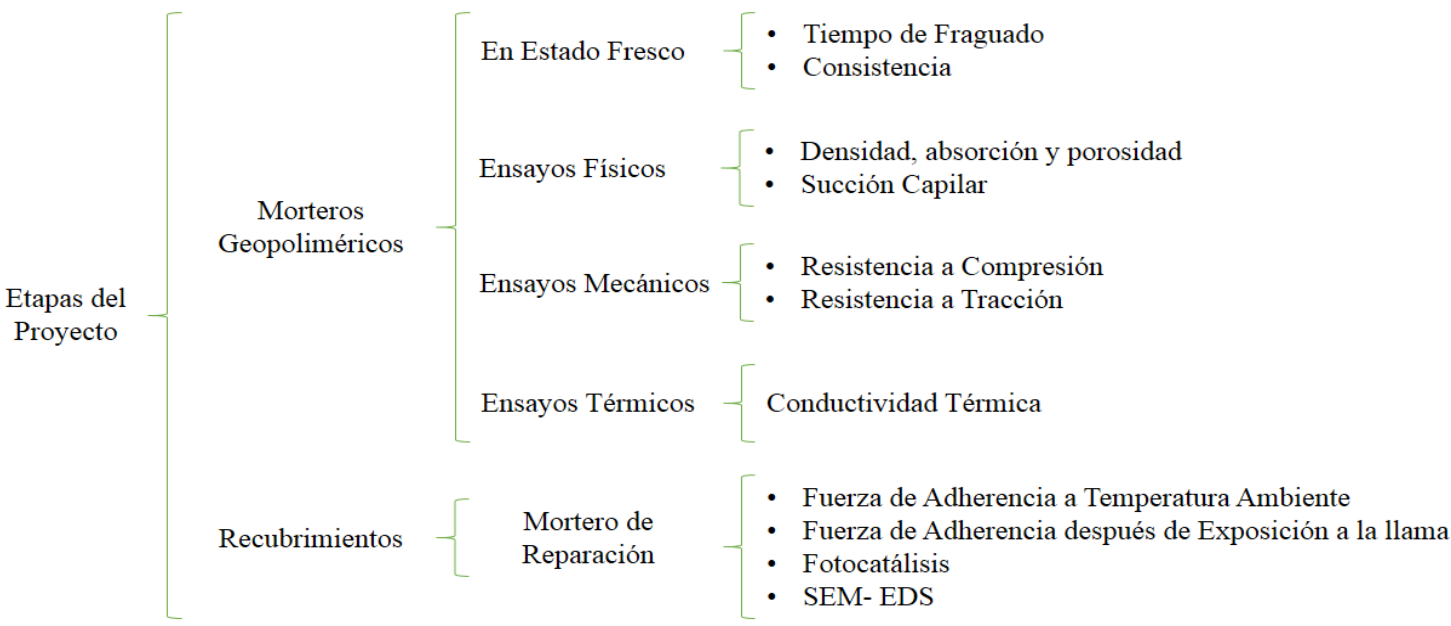

TABLA 1. CLASIFICACIÓN DE PRODUCTOS ESTRUCTURALES Y NO ESTRUCTURALES PARA REPARACIÓN DE CONCRETOS SEGÚN LA NORMA EN 1504-3

\begin{tabular}{|c|c|c|c|c|}
\hline \multirow{3}{*}{ Característica } & \multirow{2}{*}{\multicolumn{2}{|c|}{$\begin{array}{c}\text { Estructurales } \\
\text { Clase }\end{array}$}} & \multirow{2}{*}{\multicolumn{2}{|c|}{$\begin{array}{c}\text { No Estructurales } \\
\text { Clase }\end{array}$}} \\
\hline & & & & \\
\hline & R4 & R3 & $\mathbf{R} 2$ & R1 \\
\hline $\begin{array}{c}\text { Resistencia } \\
\text { compresión } \\
(\mathrm{MPa})\end{array}$ & $\geq 45$ & $\geq 25$ & $\geq 15$ & $\geq 10$ \\
\hline Adherencia & $\geq 2$ & $\geq 1,5$ & \multicolumn{2}{|c|}{$\geq 0,8$} \\
\hline $\begin{array}{c}\text { Durabilidad } \\
\text { Compatibilidad } \\
\text { Térmica }\end{array}$ & $\geq 2$ & $\geq 1,5$ & \multicolumn{2}{|c|}{$\geq 0,8$} \\
\hline $\begin{array}{l}\text { Absorción } \\
\text { Capilar } \\
\mathrm{Kg} / \mathrm{m}^{2} \cdot \mathrm{h}^{0.5}\end{array}$ & \multicolumn{2}{|c|}{$\leq 0,5$} & $\leq 0,5$ & $\begin{array}{l}\text { Ningún } \\
\text { Requisito }\end{array}$ \\
\hline
\end{tabular}

\subsection{Materiales y Caracterización}

Las materias primas empleadas en la elaboración de los morteros de reparación para estructuras de concreto fueron residuos de ladrillo (RCBW), metacaolín de origen colombiano (MK) y arena silícea, la cual se acondiciono retirando todo el material retenido en el tamiz $\mathrm{N}^{\circ} 16$ (abertura de 1,19 mm) de la clasificación ASTM. El metacaolín utilizado co- rresponde al conocido comercialmente como Fortacrete 10 y es producido por la empresa CORONA; el residuo de ladrillo fue adquirido en una empresa dedicada a la fabricación de ladrillos en la región del Valle del Cauca, el cual fue sometido a un proceso de adecuación por molienda. La composición química de los materiales (Tabla 2) se realizó utilizando un Espectrómetro de Fluorescencia de Rayos X secuencial por longitud de onda dispersiva (WDXRF) marca PANalytical modelo AXIOS mAX, equipado con un tubo de Rodio con una potencia máxima de $4.0 \mathrm{KW}$, y el tamaño y distribución de partículas mediante granulometría láser (Figura 2) en un equipo Mastersizer-2000 - Malvern Instruments, acoplado con una unidad de dispersión Hydro2000MU, en la que se aplicó agua destilada como medio de dispersión. El tamaño medio de partícula fue de $16,821 \mu \mathrm{m}$, 30,575 $\mu$ m para el MK y RCBW respectivamente.

\subsection{Síntesis de morteros}

\section{geopoliméricos}

Para la síntesis de los recubrimientos geopoliméricos se utilizaron como precursores $\mathrm{MK}$ y una mezcla binaria constituida por 10\% MK $+90 \%$ RCBW denominada MR, las cuales se activaron 
químicamente con una solución mezcla de silicato de potasio comercial $\left(12.92 \% \mathrm{~K}_{2} \mathrm{O}, 26.80 \% \mathrm{SO}_{2} \mathrm{y}\right.$ $60.28 \% \mathrm{H}_{2} \mathrm{O}$ en peso) e hidróxido de potasio (KOH). Estas mezclas fueron modificadas mediante adiciones de acronal (A), látex (L) y partículas de $\mathrm{TiO}_{2}(\mathrm{~T})$, las cuales presentan un tamaño medio de partícula de $1,59 \mu \mathrm{m}$. Se formularon cinco sistemas usando diferentes relaciones molares $\mathrm{SiO}_{2} / \mathrm{Al}_{2} \mathrm{O}_{3}(\mathrm{Si} / \mathrm{Al}), \mathrm{K}_{2} \mathrm{O}$ / $\mathrm{SiO}_{2}(\mathrm{~K} / \mathrm{S})$, liquido/solido (L/s) y relación cementante: arena (C: Ar), como se muestra en la Tabla 3. La relación liquido/solido (L/s) se ajustó de tal forma que la trabajabilidad de la mezcla permitiera su fácil aplicación.

TABLA 2. COMPOSICIÓN QUÍMICA DE LAS MATERIAS PRIMAS

\begin{tabular}{c|c|c}
\multirow{2}{*}{ Compuesto } & MK & RCBW \\
\cline { 2 - 3 } & (\% en peso) & (\% en peso) \\
\hline $\mathrm{Na}_{2} \mathrm{O}$ & 0,07 & 0,75 \\
\hline $\mathrm{MgO}$ & 0,31 & 1,36 \\
\hline $\mathrm{Al}_{2} \mathrm{O}_{3}$ & 40,63 & 23,02 \\
\hline $\mathrm{SiO}_{2}$ & 52,67 & 60,04 \\
\hline $\mathrm{P}_{2} \mathrm{O}_{5}$ & 0,03 & 0,11 \\
\hline $\mathrm{SO}_{3}$ & 0,05 & 0,13 \\
\hline $\mathrm{K}_{2} \mathrm{O}$ & 0,56 & 1,40 \\
\hline $\mathrm{CaO}$ & 0,53 & 1,15 \\
\hline $\mathrm{TiO}_{2}$ & 1,08 & 1,02 \\
\hline $\mathrm{Cr}_{2} \mathrm{O}_{3}$ & 0,02 & 0,03 \\
\hline $\mathrm{MnO}_{\mathrm{Fe}} \mathrm{O}_{3}$ & 0,01 & 0,08 \\
\hline $\mathrm{LOI}$ & 1,35 & 8,86 \\
\hline & 2,61 & 1,87 \\
\hline
\end{tabular}

TABLA 3. DOSIFICACIÓN DE LOS SISTEMAS GEOPOLIMÉRICOS

\begin{tabular}{|c|c|c|c|c|c|c|}
\hline \multirow[b]{2}{*}{$\begin{array}{c}\text { Mortero } \\
\text { Código }\end{array}$} & \multirow[b]{2}{*}{$\frac{\text { Si }}{A I}$} & \multirow[b]{2}{*}{$\frac{\mathbf{K}}{\mathbf{S i}}$} & \multirow[b]{2}{*}{ L/s } & \multirow[b]{2}{*}{ C: Ar } & \multicolumn{2}{|c|}{ Adiciones } \\
\hline & & & & & Tipo & $\begin{array}{l}\% \text { en } \\
\text { peso }\end{array}$ \\
\hline MR & \multirow{2}{*}{4} & \multirow{2}{*}{0,12} & 0,295 & \multirow{2}{*}{$1: 1,5$} & - & - \\
\hline MR-10T & & & 0,341 & & $\mathrm{TiO}_{2}$ & 10 \\
\hline MK-3A & \multirow{3}{*}{2.5} & \multirow{3}{*}{0,25} & \multirow{3}{*}{0,406} & \multirow{3}{*}{$1: 2,0$} & Acronal & 3 \\
\hline MK-5L & & & & & Latex & 5 \\
\hline MK & & & & & - & - \\
\hline
\end{tabular}

Figura 2. Distribución de tamaños de partícula para a) Metacaolín (MK) Fortacrete $10 \mathrm{y}$ b) Residuo de ladrillo (RCBW)
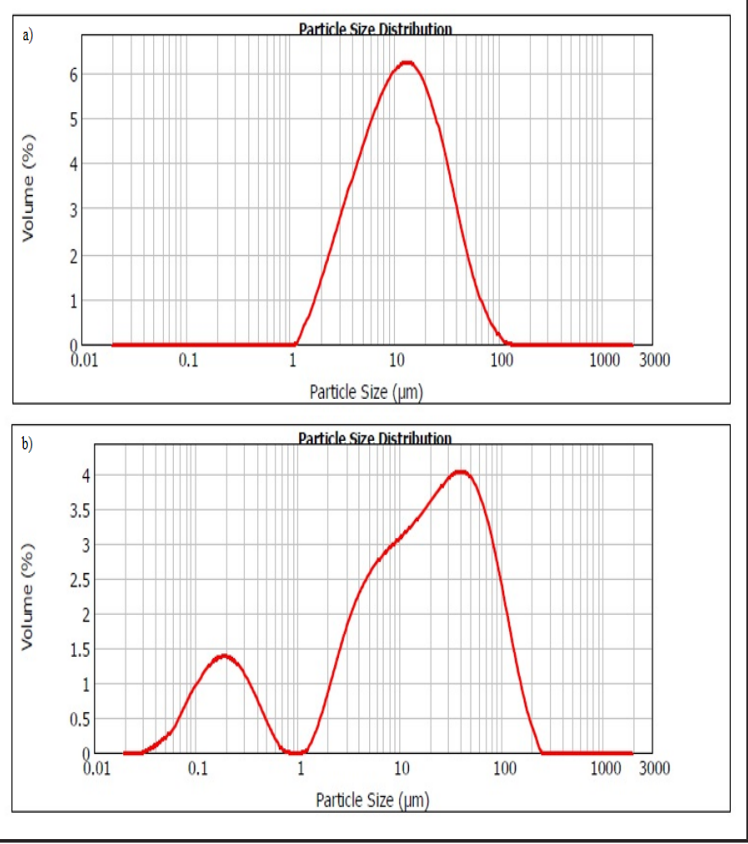

Las mezclas de mortero se prepararon en una mezcladora HOBART Vulcan 1249 utilizando diferentes etapas, inicialmente se preparó la solución activante, la cual se dejó enfriar unos minutos y posteriormente se adiciono a los precursores (MK y RCBW) previamente homogenizados, luego se adicionó lentamente la arena y la adición respectiva, finalmente se agregó agua adicional para conseguir la relación L/s establecida y se continuo la mezcla aproximadamente cinco minutos hasta conseguir una mezcla homogénea. Posteriormente, para la caracterización física y mecánica, se moldearon cubos de $2 \times 2 \mathrm{~mm}$, briquetas tipo " 8 " y cilindros de 3" de diámetro, las cuales se colocaron en una mesa vibradora eléctrica aproximadamente 20 segundos y se dejaron curar a 1 y 28 días a temperatura ambiente hasta la edad de ensayo.

\subsection{Caracterización de los materialles geopoliméricos}

Para la caracterización de los materiales geopoliméricos se realizaron los siguientes ensayos: 
- $\quad$ Ensayo de tiempo de fraguado de las pastas cementantes utilizando el método de Vicat según la norma ASTM C191-13.

- $\quad$ Ensayo de fluidez de los morteros geopoliméricos, tal como se describe en la norma ASTM C 1437-15 utilizando una mesa de flujo.

- Densidad aparente, porcentaje de absorción y porosidad a edades de curado de 28 días, bajo la norma ASTM C642 utilizando muestras cubicas de mortero

- Velocidad de absorción de agua o succión capilar de muestras cilíndricas de mortero con 28 días de curado, bajo las normas SIA 162/1 y ASTM C1585

- Resistencia a la Compresión. Para la medición de la resistencia a la compresión se moldearon 3 especímenes por cada mezcla utilizando cubos de $2 \times 2 \times 2 \mathrm{~cm}$, los cuales se dejaron curar a temperatura ambiente a edades de 1 y 28 días y posteriormente se evaluaron en una máquina universal de ensayos INSTRON 3369 con una capacidad de $50 \mathrm{kN}$ y una velocidad de deformación de $1 \mathrm{~mm}$ / min (ASTM C 109).

- Resistencia a la Tracción. Se moldearon 4 briquetas tipo ocho por cada sistema, las cuales se dejaron curar 28 días también a temperatura ambiente y se ensayaron en una máquina universal Tinius Olsen H50KS con una velocidad de $1 \mathrm{~mm} / \mathrm{min}$

- Conductividad térmica. Las propiedades térmicas se determinaron en un analizador de constantes térmicas Host Disk modelo TPS $500 \mathrm{~S}$ de Thermtest - Thermal Conductivity Instruments en un rango de trabajo de 0,03 a $100 \mathrm{~W} / \mathrm{mK}$ en conductividad térmica, por 40 segundos. Para ello se empleó un sensor Kapton 5501 con un radio de $6,403 \mathrm{~mm}$ y una resistencia de $13,15 \Omega$, y se aplicó una potencia de 956,69 mW. Se emplearon 3 pares de especímenes cúbicos de $2 \times 2 \times 2 \mathrm{~cm}$, previamente curados 28 días a temperatura ambiente.

\section{Resultados y discusión}

\subsection{Ensayo de Fluidez y tiempo de}

\section{fraguado}

En la Figura 3 se pueden observar los valores de fluidez para cada uno de los morteros empleados en la elaboración de los recubrimientos, los valores obtenidos fueron 43,0; 22,5; 56,2; 50,5 y 42,5, para los sistemas MR, MR-10T, MK, MK-3A y MK-5L, respectivamente. La medición de los diámetros de flujo se realizó cada 5 minutos durante 15 minutos para observar el cambio de trabajabilidad en el tiempo.

\section{Figura 3. Fluidez de los recubrimientos evaluados}

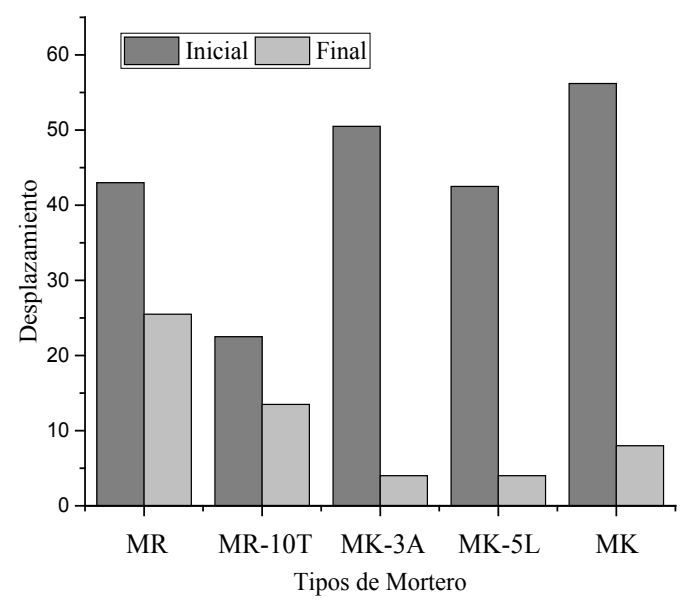

Figura 4. Tiempo de fraguado inicial y final de las pastas cementantes

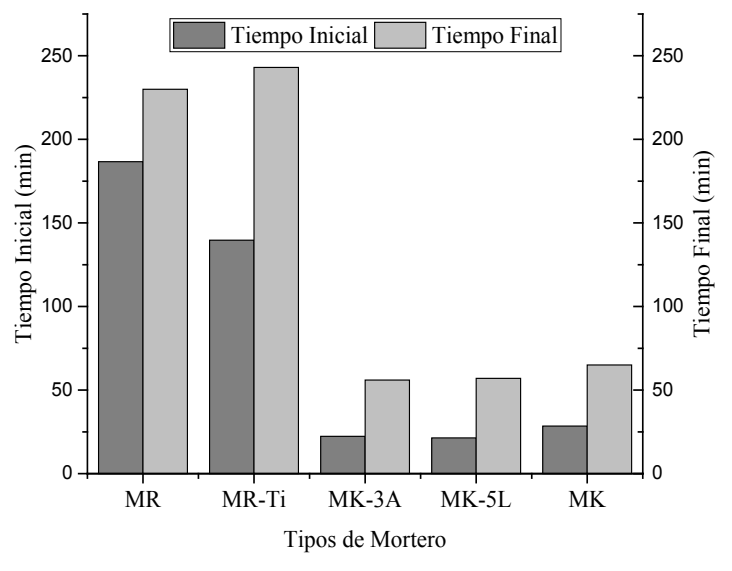


Los sistemas elaborados a base solo de MK presentan mayor fluidez en comparación a los que contienen RCBW, atribuible a la mayor relación L/s $(0,406)$. De la gráfica también se puede observar que las diferentes adiciones incorporadas en las mezclas disminuyen notablemente la fluidez de los sistemas, la adición de $\mathrm{TiO}_{2}$ disminuye en un $47,67 \%$ la fluidez, y la adición de acronal y látex en un $10,14 \%$ y $24,28 \%$ respectivamente. Aun así, cabe resaltar que, aunque los diámetros de flujo fueron bajos, la consistencia de los morteros fue adecuada para la buena aplicación del recubrimiento sobre las piezas de concreto.

En cuanto al tiempo de fraguado de las pastas geopoliméricas en la Figura 4 se aprecia que los sistemas elaborados con RCBW, es decir MR, presentan un tiempo considerablemente más elevado que los sistemas elaborados con MK, lo cual se debe principalmente a la naturaleza de los precursores y al tamaño de partícula de estos. El RCBW es un precursor semicristalino que tiene como fase principal cuarzo $\left(\mathrm{SiO}_{2}\right)$ y un tamaño medio de partícula de 30,575 $\mu \mathrm{m}$, mientras que el MK es un material que presenta una estructura altamente amorfa proveniente de su proceso de obtención, lo cual lo hace altamente reactivo en comparación a otros precursores y además presenta un tamaño medio de partícula de $16,821 \mu$ m. En el caso del sistema MR-10T la disminución de los tiempos de fraguado, se puede deber a su reducido tamaño de partícula y elevada superficie específica a diferencia del precursor, lo cual hace que estas adiciones se conviertan en puntos de nucleación, permitiendo una mayor velocidad de reacción. Al respecto, Duan et al. (2016) afirman que la adición de polvo de $\mathrm{TiO}_{2}$ acelera significativamente la tasa de hidratación y promueve el grado de hidratación de los materiales cementantes a edades tempranas.

Lee, Kim and Lee (2016) han reportado que adiciones orgánicas como el látex y sus derivados pueden retardar el fraguado de los geopolímeros debido a que estas reducen el pH en la matriz, lo que retrasaría químicamente el proceso de polimerización. Sin embargo, en este estudio no se evidencio tal fenómeno en los sistemas MK-3A y MK-5L, lo cual podría deberse a la forma de mezclado, ya que primero se activaron los precursores y posterior a ello se adiciono el acronal y látex, evitando así perturbar físicamente la reacción entre las partículas y el activador alcalino.

\subsection{Propiedades mecánicas: Resis- tencia a la compresión y tracción}

En la Figura 5 se presentan los resultados de los ensayos de resistencia a la compresión y tracción de los materiales geopoliméricos. En la Figura 5a) se aprecia que el sistema MR presenta la mayor resistencia a la compresión a los 28 días de curado alcanzando un valor de 16,76 $\mathrm{MPa}$, seguido del sistema MK-5L con un valor de 13,64 MPa. El desarrollo de la resistencia inicial en los morteros basados en MK fue notable en comparación a los sistemas MR, ya que a 1 día de curado se obtuvo más de $7 \mathrm{MPa}$, lo cual puede atribuirse a la alta reactividad del MK debido a su alto contenido de $\mathrm{Al}_{2} \mathrm{O}_{3}$ reactivo y su carácter amorfo a diferencia del RCBW. Los residuos de ladrillos de arcilla roja (RCBW), son materiales que en el proceso de obtención han sido expuestos a altas temperaturas, en ocasiones sin un control apropiado, lo cual, puede ocasionar una disminución en su reactividad (Robayo et al., 2016). Además, cabe resaltar que la disolución de los aluminosilicatos se da con mayor dificultad en solución de $\mathrm{KOH}$ que en $\mathrm{NaOH}$, debido a que los iones $\mathrm{Na}^{+}$son más pequeños que los iones $\mathrm{K}^{+}$, lo cual permite establecer fácilmente enlaces con el anión silicato, dando lugar a pequeños oligómeros (Liew et al., 2016), (Xu and Van Deventer, 2000).

En cuanto a los sistemas basados solo en MK, puede observarse que MK-5L presenta la mayor resistencia entre ellos (13,63 MPa), lo cual puede atribuirse al contenido de látex, ya que autores como Lee, Kim and Lee, (2016) mencionan que la incorporación de mezclas orgánicas como caucho de copolímero de estireno butadieno, acetato de polivinilo, polímero de acrilato, entre otros, en materiales cementantes dan lugar a mejoras en resistencia a la 
compresión y tenacidad, debido a la disminución de la presencia de microgrietas. El sistema MK-3A por su parte presento una menor resistencia mecánica lo cual se atribuye a que el acronal comercial utilizado en la investigación presenta altos contenidos de agua y modifica la relación L/s usada en la mezcla.

La mayor resistencia del sistema MR a 28 días de curado puede ser atribuida a la menor relación L/s empleada en la mezcla $(0,295)$; ya que, al igual que ocurre en los cementos portland con el incremento de la relación agua/cementante, en los geopolimeros la relación L/s puede afectar significativamente las propiedades mecánicas del material (Mu et al., 2017). Igualmente, cabe anotar que la resistencia a la compresión de los geopolímeros puede verse fuertemente influenciada por las relaciones $\mathrm{Si} / \mathrm{Al}$ y $\mathrm{K} / \mathrm{Si}$ utilizadas en la mezcla, por ejemplo, al utilizar bajo contenido de álcali $\left(\mathrm{K}^{+}\right)$habrá cantidades insuficientes de $\mathrm{OH}^{-}$para disolver el $\mathrm{Si}^{4+} \mathrm{y} \mathrm{Al}^{3+}$ de los aluminosilicatos e insuficiente álcali para permitir la geopolimerización completa, por lo tanto, el material será de estructura poco densa y porosa (Zhuang et al., 2016). En cuanto a la resistencia a la tracción, en la Figura 5 b) se evidencia una relación directa con la resistencia a la compresión, ya que se presenta un comportamiento similar. El sistema MR presentó la mayor resistencia a la tracción con un valor de 2,11 $\mathrm{MPa}$, seguido del sistema MK con un valor de 1,51 MPa.

\subsection{Propiedades Físicas: Absorción y} Porosidad

Otras características físicas de los morteros geopoliméricos son la densidad y los porcentajes de absorción de agua y porosidad, los cuales pueden ser observados en la Figura 6 a) y b), respectivamente. En la Figura 6 a) puede verse que los morteros de mayor densidad son MK-5L y MK con valores de 2289 y $2680 \mathrm{Kg} / \mathrm{m}^{3}$, respectivamente, los cuales presentan a su vez los valores de porosidad más elevados de $25,49 \%$ y 29,56 \% (Figura 6b). Dicho comportamiento se presentó debido a que estos poseen mayor cantidad de arena en su composición, el cual es un material denso y no debido propiamente a la naturaleza de los productos de reacción (Mermerdaş et al., 2017). La incorporación del $\mathrm{TiO}_{2}$ a MR (MR-10T) incremento los valores de absorción y porosidad, y aunque las partículas ultrafinas deberían actuar como agentes de relleno disminuyendo el porcentaje de poros, en este sistema se empleó una relación L/s mayor a la de MR debido a la mayor demanda de agua. Cabe aclarar, que los resultados obtenidos, en términos generales, están relacionados con el desempeño mecánico de las mezclas (Figura 5).

Figura 5. a) Resistencia a la compresión y b) Resistencia a la tracción de los morteros
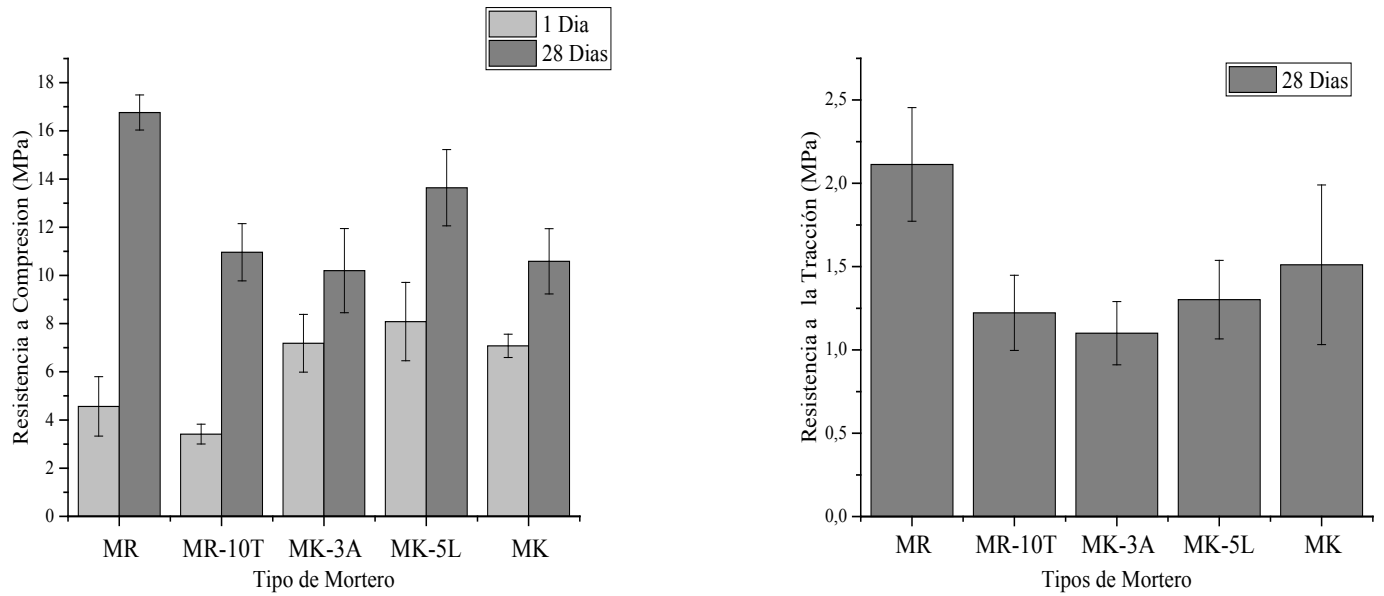
Figura 6. a) Densidad, b) Porcentaje de absorción y porosidad de los sistemas geopoliméricos evaluados
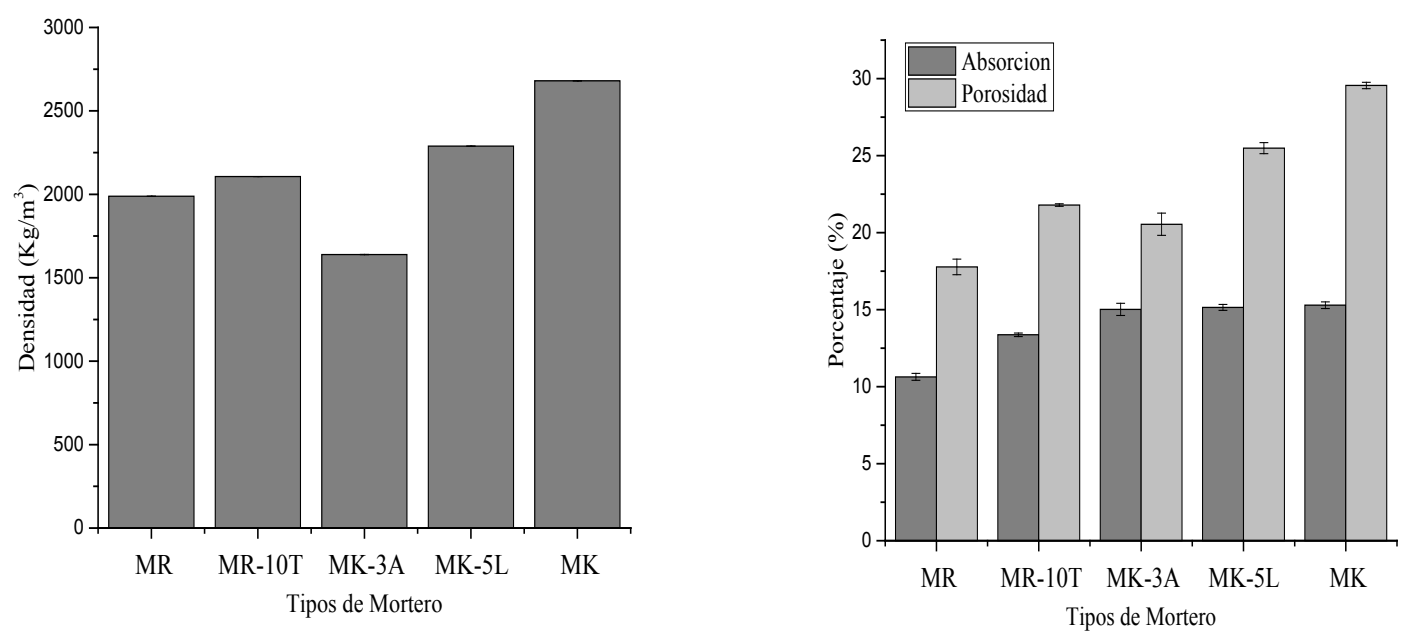

Un ensayo complementario realizado fue el de succión capilar para determinar la velocidad de absorción de agua de los morteros geopoliméricos endurecidos a 28 días, lo cual permite hallar la intensidad de absorción (I) en función del tiempo como se puede observar en la Figura 7. A partir de estas curvas es posible calcular las velocidades de absorción inicial $(\mathrm{K})$ y secundaria $\left(\mathrm{K}_{2}\right)$, las cuales permiten conocer el tiempo de saturación (T) de los morteros, a partir de lo cual se calcula la resistencia a la penetración del agua (m) y finalmente la porosidad efectiva $(\varepsilon)$ de cada muestra. Se puede observar en la Figura 7 que la velocidad de absorción más alta corresponde al sistema MK, mientras que MR presentó la velocidad más baja con valores de 0,0631 y $0,04166 \mathrm{Kg} / \mathrm{m}^{2} . \mathrm{s}^{1 / 2}$, respectivamente. Esto concuerda con los porcentajes de poros permeables mencionados anteriormente y con la porosidad efectiva o cantidad de poros interconectados (capilares) pues este factor es mayor también en MK comparado a MR, 39,35 y 24,22 \% respectivamente. Por otro lado, es evidente que las adiciones de acronal y látex al sistema MK lograron impermeabilizar un poco las muestras ya que MK-3A y MK-5L presentaron velocidades $(\mathrm{K})$ y porosidad efectiva menores con valores de 0,04298 y $0,04019 \mathrm{Kg} / \mathrm{m}^{2} \cdot \mathrm{s}^{1 / 2}$ y 24,12 y $22,76 \%$, respectivamente; lo cual es un comporta- miento esperado, ya que estas sustancias son copolímeros basados en acetato de polivinilo (PVA) especialmente diseñados para sellar superficies.

Figura 7. Cinética de succión capilar de los sistemas geopoliméricos evaluados

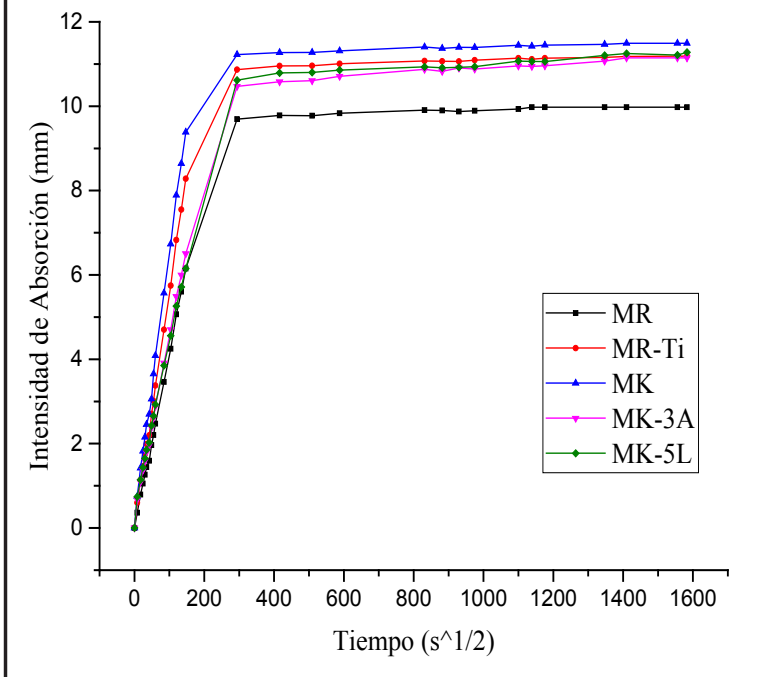

3.4. Propiedades Térmicas: Conductividad Térmica

En la Tabla 4 pueden observarse las constantes térmicas obtenidas para cada uno de los sistemas geopoliméricos estudiados, los cuales se compararon 
con un mortero de cemento convencional (OPC) diseñado con una relación a/c y una relación C: Ar de 0,47 y $1: 2$, respectivamente, con un valor de desplazamiento en una mesa de flujo de 44; característica de fluidez similar a la de las mezclas geopolíméricas. Los valores de conductividad térmica varían de 0,720 a $1,116 \mathrm{~W} / \mathrm{m}^{\circ} \mathrm{K}$, la difusividad térmica por su parte se encuentra en un rango de 0,381 a $0,605 \mathrm{~m}^{2} / \mathrm{s}$ y los valores de calor especifico varían de 0,929 a 2,728 $\mathrm{mJ} / \mathrm{m}^{3 \circ} \mathbf{K}$ Villaquirán-Caicedo et al., (2015) reportan valores de difusividad entre $0.16-0.27 \mathrm{~mm}^{2} / \mathrm{s}$ y valores de conductividad de $0.35 \mathrm{~W} / \mathrm{m} \cdot \mathrm{K}$ para pastas de $\mathrm{MK}$ activado con $\mathrm{KOH}$ y $\mathrm{K}_{2} \mathrm{SiO}_{3}$, con relación $\mathrm{Si} /$ $\mathrm{Al}=2.5$, sin embargo aunque se utilizan materiales $\mathrm{y}$ dosificaciones similares, no es posible comparar con sus resultados debido a que en el presente estudio se utilizaron morteros y no pastas.

TABLA 4. CONSTANTES TÉRMICAS PARA LOS RECUBRIMIENTOS GEOPOLIMÉRICOS EVALUADOS A 28 DÍAS DE CURADO

\begin{tabular}{c|c|c|c|} 
Mortero & $\begin{array}{c}\text { Conductividad } \\
\text { Térmica }(\mathrm{W} / \\
\left.\mathrm{m}^{\circ} \mathrm{K}\right)\end{array}$ & $\begin{array}{c}\text { Difusividad } \\
\text { Térmica } \\
\left(\mathrm{m}^{2} / \mathrm{s}\right)\end{array}$ & $\begin{array}{c}\text { Calor } \\
\text { Especifico } \\
\left(\mathrm{mJ}^{3} \mathrm{~m}^{30} \mathrm{~K}\right)\end{array}$ \\
\hline MR & 0,817 & 0,605 & 0,929 \\
\hline MR-10T & 0,932 & 0,402 & 2,319 \\
\hline MK-3A & 0,629 & 0,399 & 1,578 \\
\hline MK-5L & 0,745 & 0,437 & 1,703 \\
\hline MK & 0,720 & 0,381 & 1,888 \\
\hline OPC & 1,116 & 0,410 & 2,728 \\
\hline
\end{tabular}

Generalmente, las constantes térmicas como la conductividad térmica, la difusividad y el calor específico se relacionan con la porosidad del material, su distribución e interconectividad. Así como también el orden de corto alcance de los productos de reacción y su composición química nominal. Todos estos factores afectan las propiedades de transporte de calor de los geles de geopolímero. Los sistemas basados solo en MK presentan los valores de conductividad más bajos, lo cual concuerda con la literatura, pues como se observó en las Figuras 5 y $\mathbf{6}$, son los sistemas con más altos porcentajes de poros permeables y porosidad efectiva, es decir, mayor cantidad de poros interconectados. Por lo tanto, se establece una relación inversamente proporcional entre estos factores.

Los sistemas MR y MR-10T presentan los menores porcentajes de poros permeables y porosidad efectiva, sin embargo, presentan valores de conductividad más altos, lo que se puede atribuir principalmente a su composición química ya que el precursor mayoritario en su elaboración fue RCBW, este material posee porcentajes significativos de óxidos de hierro $\left(\mathrm{Fe}_{2} \mathrm{O}_{3}\right)$, conocido como un material con buenas propiedades térmicas. Por otro lado, se ha mencionado que el aumento de la relación $\mathrm{Si} / \mathrm{Al}$ en los geopolímeros aumenta linealmente la conductividad térmica, ya que se contribuye a una reacción de policondensación mejor con una disminución en los poros permeables y una densificación mayor del material, lo que por consiguiente aumenta la difusividad térmica y el calor especifico, ya que se da una transferencia de energía más eficiente (VillaquiránCaicedo et al., 2015).

Al comparar las constantes térmicas de los sistemas geopoliméricos evaluados con las de un mortero de OPC (Tabla 4), se evidenciaron valores mayores de conductividad en este último $(1,116 \mathrm{~W} /$ $\mathrm{m}^{\circ} \mathrm{K}$ ), al igual que de difusividad y calor especifico, lo cual es un resultado muy positivo ya que permite considerar los materiales evaluados para su aplicación como recubrimientos aislantes.

\section{Conclusiones}

De los resultados obtenidos en el presente estudio se puede extraer las siguientes conclusiones:

- Las diferentes adiciones incorporadas en las mezclas disminuyen notablemente la fluidez de los sistemas y su tiempo de fraguado. La adición de $10 \% \mathrm{TiO}_{2}$ disminuye en un $47.67 \%$ la fluidez, así como la adición de 3\% acronal y 5\% látex la disminuyen en un $10.14 \%$ y $24.28 \%$. El tiempo de fraguado se ve afectado negativamente hasta un $12.31 \%$ y $13.85 \%$ al adicionar látex y acronal al sistema MK, respectivamente. 
- Es posible elaborar morteros geopoliméricos a partir de RCBW y MK de origen nacional, con resistencias a la compresión de 16.76, 10.96, $10.20,13.64$ y $10.59 \mathrm{MPa}$, para los sistemas MR, MR-10T, MK-3A, MK-5L y MK, respectivamente. Así como también resistencias a la tracción de 2.113, 1.223, 1.101, 1.302 y $1.511 \mathrm{MPa}$, para los mismos sistemas, respectivamente. Lo cual permite clasificarlos como morteros de reparación no estructurales Clase R1 (Resistencia a compresión $\geq 10 \mathrm{MPa}$ ) y R2 (Resistencia a compresión $\geq 15 \mathrm{MPa}$ ), en concordancia con la norma EN 1504-3.

- Los valores de absorción o succión capilar obtenidos para los sistemas MR, MR-10T, MK-3A, MK-5L y MK $(0.757,0.826,1.861,1.666$ y 0.754 $\mathrm{Kg} / \mathrm{m}^{2} \cdot \mathrm{h}^{1 / 2}$ respectivamente), no cumplen la norma EN 1504-3 para morteros Clase R2. Sin embargo, para la Clase R1 no existe ningún requisito de absorción capilar, lo que permite clasificar el mortero MK-5L como material de reparación Clase R1con base en esta propiedad. Ajustes en el diseño de la matriz podría reducir notablemente estos valores ampliando el campo de aplicación de los materiales evaluados.

- $\quad$ Se evidenció que los sistemas geopoliméricos MR, MR-10T, MK-3A, MK-5L y MK presentaron menor conductividad térmica que un mortero convencional de OPC con las mismas características, lo que sugiere su uso como recubrimientos de tipo aislante.

- Finalmente, para dar cumplimiento a las normas y hacer una clasificación más ajustada se requiere avanzar en la evaluación de la adherencia y durabilidad de los recubrimientos en sustratos de concreto.

\section{Referencias}

Duan, P.; Yan, C.; Luo, W.; Zhou, W. (2016). Effects of adding nano- $\mathrm{TiO}_{2}$ on compressive strength, drying shrinkage, carbonation and microstructure of fluidized bed fly ash based geopolymer paste. Constr. Build.
Mater., 106, pp. 115-125. doi: 10.1016/j.conbuildmat.2015.12.095.

Irfan Khan, M.; Azizli, K.; Sufian, S.;Man, Z. (2014). Effect of $\mathrm{Na} / \mathrm{Al}$ and $\mathrm{Si} / \mathrm{Al}$ Ratios on Adhesion Strength of Geopolymers as Coating Material. Appl. Mech. Mater., 625, pp. 85-89. doi: 10.4028/www.scientific. net/AMM.625.85.

Irfan Khan, M.; Azizli, K.; Sufian, S.;Man, Z. (2015). Sodium silicate-free geopolymers as coating materials: Effects of $\mathrm{Na} / \mathrm{Al}$ and water/solid ratios on adhesion strength. Ceram. Int., 41(2), pp. 2794-2805. doi: 10.4028/www.scientific.net/AMM.625.85.

Krivenko, P.V.; Guzii, S.G.; Bodnarova, L.; Valek, J.; Hela, R.; Zach, J. (2016). Effect of thickness of the intumescent alkali aluminosilicate coating on temperature distribution in reinforced concrete. J. Build. Eng., 8, pp. 14-19. doi: 10.1016/j.jobe.2016.09.003.

Lee, N.K.; Kim, E.M.; Lee, H.K. (2016). Mechanical properties and setting characteristics of geopolymer mortar using styrene-butadiene (SB) latex. Constr. Build. Mater., 113, pp. 264-272. doi: 10.1016/j.conbuildmat.2016.03.055.

Liew, Y.M.; Heah, C.Y; Mohd Mustafa, A.B.; Kamarudin, H. (2016). Structure and properties of clay-based geopolymer cements: A review. Prog. Mater. Sci., 83, pp. 595-629. doi: 10.1016/j.pmatsci.2016.08.002.

Mermerdaș, K.; Manguri, S.; Nassani, D.E.; Oleiwi, S.M. (2017). Effect of aggregate properties on the mechanical and absorption characteristics of geopolymer mortar.Eng. Sci. Technol. an Int. J., 20(6), pp. 1642-1652. doi: 10.1016/j.jestch.2017.11.009.

Mu, S.; Liu, J.; Lin, W.; Wang, Y.; Liu, J.; Shi, L.; Jiang, Q. (2017). Property and microstructure of aluminosilicate inorganic coating for concrete: Role of water to solid ratio. Constr. Build. Mater., 148, pp. 846-856. doi: 10.1016/j.conbuildmat.2017.05.070.

Pacheco-Torgal, F.; Abdollahnejad, Z.; Miraldo, S.; Baklouti, S.; Ding, Y. (2012). An overview on the potential of geopolymers for concrete infrastructure rehabilitation. Constr. Build. Mater., 36, pp. 1053-1058. doi: 10.1016/j.conbuildmat.2012.07.003.

Robayo, R.A.; Mulford, A.; Munera, J.; and Mejía de Gutiérrez, R. (2016). Alternative cements based on alkali-activated red clay brick waste. Constr. Build. Mater., 128, pp. 163-169. doi: 10.1016/j.conbuildmat.2016.10.023. 
Tamburini, S.; Natali, M.; Garbin, E.; Panizza, M.; Favaro, M.; Valluzzi, M.R. (2017). Geopolymer matrix for fibre reinforced composites aimed at strengthening masonry structures. Constr. Build. Mater., 141, pp. 542-552. doi: 10.1016/j.conbuildmat.2017.03.017.

Vejmelková, E.; Koňáková, D.; Čáchová, M.; Keppert, M.; Černý, R. (2012). Effect of hydrophobization on the properties of lime-metakaolin plasters. Constr. Build. Mater., 37, pp. 556-561. doi: 10.1016/j. conbuildmat.2012.07.097.

Villaquirán-Caicedo, M.A.; M. de Gutiérrez, R.; Sulekar, S.; Davis, C.; Nino, J.C. (2015). Thermal properties of novel binary geopolymers based on metakaolin and alternative silica sources. Appl. Clay Sci., 118, pp. 276-282. doi: 10.1016/j.clay.2015.10.005.

Wiyono, D.; Antoni; Hardjito, D. (2015). Improving the Durability of Pozzolan Concrete Using Alkaline Solution and Geopolymer Coating. Procedia Eng., 125, pp. 747-753. doi: 10.1016/j.proeng.2015.11.121.

$\mathrm{Xu}$, H.; Van Deventer, J.S J. (2000). The geopolymerisation of alumino-silicate minerals. Int. J. Miner. Process., 59(3), pp. 247-266. doi: 10.1016/S03017516(99)00074-5.

Zhang, Z.; Wang, K.; Mo, B.; Li, X.; Cui, X.(2015). Preparation and characterization of a reflective and heat insulative coating based on geopolymers. Energy Build., 87, pp. 220-225. doi: 10.1016/j.enbuild.2014.11.028.

Zhuang, X.Y.; Chen, L.; Komarneni, S.; Zhou, C.H.; Tong, D.S.; Yang, H.M.; Yu, W H.; Wang, H. (2016). Fly ash-based geopolymer: clean production, properties and applications. J. Clean. Prod., 125, pp. 253-267. doi: 10.1016/j.jclepro.2016.03.019. 